\title{
Cytotoxic Lesion in the Splenium of Corpus Callosum Secondary to Subacute Methotrexate Neurotoxicity
}

\author{
Ahmad A. Al-Awwad ${ }^{1, \odot ~ A h m e d ~ K o r i e s h ² ~}$ \\ ${ }^{1}$ Department of Neurology, University of Oklahoma Health Sciences \\ Center, Oklahoma City, Oklahoma, United States \\ 2Department of Neurology, University of Missouri, Columbia, \\ Missouri, United States
}

\begin{abstract}
Address for correspondence Ahmad A. Al-Awwad, MD, Neurology University of Oklahoma Health Sciences Center, Oklahoma, United States (e-mail: a.alawwad@yahoo.com).
\end{abstract}

Avicenna J. Med. 2021;3:160-162.

\begin{abstract}
Keywords

- methotrexate

neurotoxicity

- splenium lesion

- dextromethorphan

Methotrexate neurotoxicity can present with a wide spectrum of neurologic symptoms and brain magnetic resonance imaging (MRI) typically demonstrates cerebral edema, demyelination, multifocal white matter necrosis, and atrophy relatively selective for the deep cerebral white matter. Here, we report a case of subacute methotrexate neurotoxicity in a 40-year-old man with B cell acute lymphoblastic leukemia. Brain MRI showed cytotoxic lesion in the splenium of corpus callosum and left middle cerebellar peduncle. Patient significantly improved 24 hours after receiving oral dextromethorphan. Methotrexate neurotoxicity should be suspected in any symptomatic patient receiving high dose of methotrexate or intrathecal methotrexate therapy. Dextromethorphan should be considered in these patients as it can modulate the excitatory responses to homocysteine and its metabolite which are usually elevated in such patients.
\end{abstract}

\section{Introduction}

Methotrexate (formerly amethopterin) is an antimetabolite used in the treatment of severe psoriasis, adult rheumatoid arthritis, and certain neoplastic diseases including acute lymphoblastic leukemia (ALL). ${ }^{1}$ Methotrexate neurotoxicity (MNT) is a known adverse reaction that happens likely through disruption of central nervous system (CNS) folate homeostasis and/or direct neuronal damage. Brain magnetic resonance imaging (MRI) typically reveals diffusion restriction abnormalities with pathologic findings including cerebral edema, demyelination, multifocal white matter necrosis, and atrophy relatively selective for the deep cerebral white matter. ${ }^{2,3}$

\section{Case Description}

A 40-year-old man with B cell ALL diagnosed 3 months prior to admission currently on consolidation therapy with

published online

August 4, 2021
DOI https://doi.org/

$10.1055 / \mathrm{s}-0041-1732486$

ISSN 2231-0770 pegaspargase and vincristine was admitted for management of neutropenic fever, severe holocranial headache, nausea, and encephalopathy. Physical exam on admission was remarkable for fever at 38.4 and low Glasgow Coma Scale of 11 with nonfocal neurologic exam. White blood count was low at 980 cell per cubic microliter with absolute neutrophil count of 350 cell per cubic microliter (normal $>4,000$ and $>1,500$, respectively). He was started on broad spectrum antibiotics and extensive infectious work-up was sent. He was also given filgrastim for severe neutropenia. Brain MRI with contrast did not show any abnormal contrast enhancement; however, it revealed diffusion restriction on diffusion-weighted imaging (DWI) sequence with correlating low signal on apparent diffusion coefficient (ADC) sequence at the splenium of corpus callosum as well as a small focus within the left middle cerebellar peduncle ( - Fig. 1 ). Electroencephalogram, toxic metabolic panel, cytology, bacterial, and viral studies in serum and cerebrospinal fluid (CSF) were all unremarkable. Nevertheless, the patient's mental status did not improve, in 

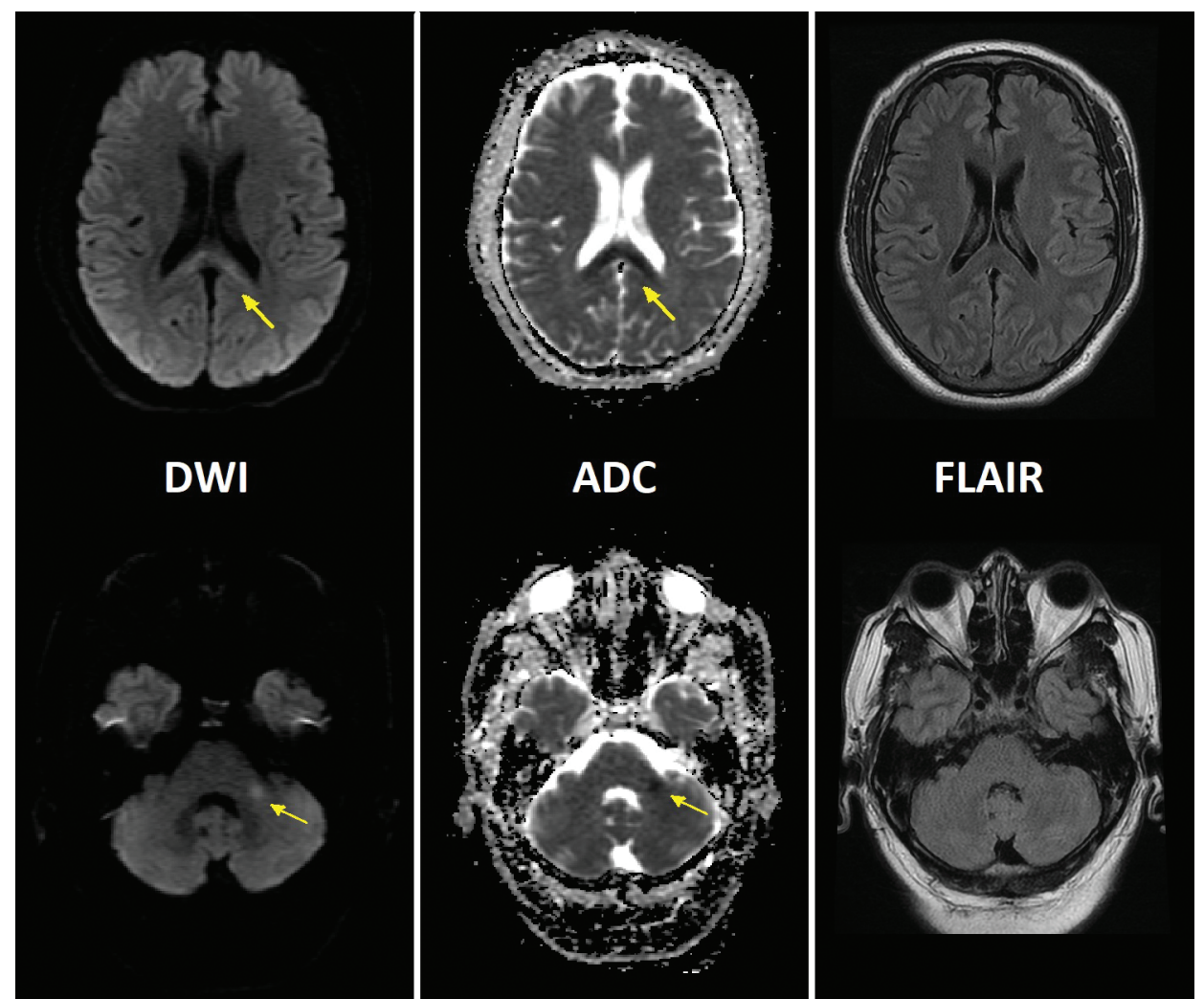

Fig. 1 Magnetic resonance imaging (MRI) shows axial diffusion-weighted imaging (DWI), apparent diffusion coefficient (ADC), and fluid-attenuated inversion recovery (FLAIR) sequences. Yellow arrows point to increased diffusion restriction with ADC correlation in the splenium of the corpus callosum (upper row) and left middle cerebellar peduncle (lower row). There are no corresponding changes on FLAIR in either cut.

spite of supportive treatment. Fever and neutropenia both resolved on day 4 of admission; however, neurologic exam remains unchanged.

As patient received three doses of $15 \mathrm{mg}$ intrathecal methotrexate for ALL (last dose was 14 days prior to admission), subacute MNT was suspected. On day 6 of admission, dextromethorphan $60 \mathrm{mg}$ every 12 hours was empirically started and the next day patient significantly improved and his neurologic exam was back to normal. He was monitored for another 24 hours then discharged home in good condition with total of 7 days of oral dextromethorphan $60 \mathrm{mg}$ twice daily. Patient did not have any recurrent of his symptoms upon 1-month follow-up.

\section{Discussion}

MNT is a well-known side effect that presents most frequently after prolonged low-dose oral, intrathecal, or high-dose methotrexate. It has been categorized as being acute, subacute, or chronic. ${ }^{4,5}$ Acute MNT occurs within a few hours after methotrexate administration and patients usually exhibit signs of chemical meningitis including fever, somnolence, confusion, headache, nausea, vomiting, and dizziness. Subacute MNT is observed within days to weeks of methotrexate therapy where patient usually exhibit seizures or stroke-like symptoms including hemiparesis, hemisensory deficits, aphasia, and diplopia and some patients may experience fever and encephalopathy (similar to what was described in our case). ${ }^{6}$ Chronic CNS toxicity occurs months to years after methotrexate therapy where patients develop cognitive dysfunction, behavioral abnormalities, and spasticity. Between all different types of MNT, the subacute form stands out as the most challenging one to be diagnosed. The association between sudden onset and acute progression of symptoms (frequently similar to the presentation of stroke) along with receiving methotrexate days or even weeks prior can be easily missed. Hence, MNT should be always on the differential for any patient with unexplained neurologic symptoms who received methotrexate therapy.

The pathogenesis of MNT is multifactorial, but CNS folate homeostasis likely plays a major role in it. Some studies suggested that acute toxicity is partly mediated by adenosine as methotrexate induces metabolic changes leading to increased extracellular adenosine. ${ }^{7}$ Subacute and chronic toxicity, on the other hand, are probably mediated with homocysteine, S-adenosylmethionine/S-adenosylhomocysteine, and other excitatory amino acids. ${ }^{8}$ There is usually an elevated level of homocysteine in CSF of patients who had received methotrexate. ${ }^{6,9}$ Homocysteine is directly toxic to vascular endothelium and its metabolites are excitatory agonists of the N-methyl-D-aspartate (NMDA) receptor. ${ }^{10}$ Dextromethorphan, a noncompetitive NMDA receptor antagonist, can modulate the excitatory responses to homocysteine and its metabolites and hence it has been used as 
a treatment of the neurological dysfunction associated with MNT and in some centers it is used as a prophylactic agent prior to methotrexate administration., ${ }^{6,10,11}$

Our patient's MRI showed diffusion restriction on DWI with ADC correlation at the splenium of corpus callosum and the left middle cerebellar peduncle. These changes are consistent with cytotoxic edema. Cytotoxic cerebral edema refers to a type of cerebral edema in which water is trapped inside the cells resulting in their swelling. This intracellular edema mainly affects gray matter but also involves the white matter as astrocytes are also involved..$^{12}$ As cells swell due to inward shift of water, this will lead into decrease in diffusion, which will be identified as high signal on DWI and low signal on ADC. ${ }^{12}$ Glutamate, a known neurotransmitter, plays major role in excitotoxicity by acting on aquaporins and NMDA receptors (among other receptors) leading into influx of water into both astrocytes and neurons resulting in cytotoxic edema appearance on MRI. ${ }^{13}$ The elevated levels of homocysteine and its metabolites in patients treated with methotrexate can act as an excitatory agonists of NMDA receptors which can possibly explain the cytotoxic edema seen in such patients. The neurons, astrocytes, and oligodendrocytes of the corpus callosum have a higher density of receptors, including cytokine receptors, toxin receptors, and drug receptors comparing with other parts of the brain which makes the corpus callosum, and particularly the splenium, more vulnerable for cytotoxic edema. ${ }^{13,14}$

Financial Support

None.

Statement of Informed Consent

Informed consent to publish this case was obtained from the patient and his wife.

Conflict of Interest

None declared.

\section{References}

1 FDA methotrexate label. Available at: https://www.accessdata.fda.gov/drugsatfda_docs/label/2016/008085s066lbl.pdf. Accessed June 9, 2021
2 Rollins N, Winick N, Bash R, Booth T. Acute methotrexate neurotoxicity: findings on diffusion-weighted imaging and correlation with clinical outcome. AJNR Am J Neuroradiol 2004;25(10):1688-1695

3 Ebner F, Ranner G, Slavc I, et al. MR findings in methotrexate-induced CNS abnormalities. AJNR Am J Neuroradiol 1989;10(5):959-964

4 Smith B. Brain damage after intrathecal methotrexate. J Neurol Neurosurg Psychiatry 1975;38(8):810-815

5 Afshar M, Birnbaum D, Golden C. Review of dextromethorphan administration in 18 patients with subacute methotrexate central nervous system toxicity. Pediatr Neurol 2014;50(6):625-629

6 Boogerd W, vd Sande JJ, Moffie D. Acute fever and delayed leukoencephalopathy following low dose intraventricular methotrexate.J Neurol Neurosurg Psychiatry 1988;51(10):1277-1283

7 Chan ES, Cronstein BN. Molecular action of methotrexate in inflammatory diseases. Arthritis Res 2002;4(4):266-273

8 Vezmar S, Becker A, Bode U, Jaehde U. Biochemical and clinical aspects of methotrexate neurotoxicity. Chemotherapy 2003;49(1-2):92-104

9 Kishi S, Griener J, Cheng C, et al. Homocysteine, pharmacogenetics, and neurotoxicity in children with leukemia. J Clin Oncol 2003;21(16):3084-3091

10 Drachtman RA, Cole PD, Golden CB, et al. Dextromethorphan is effective in the treatment of subacute methotrexate neurotoxicity. Pediatr Hematol Oncol 2002;19(5):319-327

11 Fustino NJ, Juhl K, Leister J. Dextromethorphan administration on day 0 and day 7 for secondary prevention of methotrexate-induced neurotoxicity in childhood acute lymphoblastic leukemia: a retrospective case series. J Pediatr Hematol Oncol 2020;doi:10.1097/MPH.0000000000001714

12 von KummerR, Dzialowski I. Imaging of cerebral ischemicedema and neuronal death. Neuroradiology 2017;59(6):545-553

13 Starkey J, Kobayashi N, Numaguchi Y, Moritani T. Cytotoxic lesions of the corpus callosum that show restricted diffusion: mechanisms, causes, and manifestations. Radiographics 2017;37(2):562-576

14 Goursaud S, Kozlova EN, Maloteaux JM, Hermans E. Cultured astrocytes derived from corpus callosum or cortical grey matter show distinct glutamate handling properties. J Neurochem 2009;108(6):1442-1452 